\title{
Análisis de la cadena de comercialización del chile habanero de Yucatán y su denominación de origen *
}

\author{
Analysis of the Supply Chain of Yucatán Habanero Pepper and its Designation \\ of Origin
}

\author{
José Apolinar Zapata-Aguilar \\ Doctor en Análisis Estratégico y Desarrollo Sustentable, Universidad Tecnológica Metropolitana, \\ Mérida-México, jose.zapata@utmetropolitana.edu.mx \\ Pablo Pérez-Akaki \\ Doctor en Geografía Económica, Universidad Nacional Autónoma de México, \\ Naucalpan de Juárez - México, ppablo@apolo.acatlan.unam.mx \\ Carlos Antonio Moo-Novelo \\ Maestro en Gobierno y Políticas Públicas, Universidad Tecnológica Metropolitana, \\ Mérida-México, carlos.moo@utmetropolitana.edu.mx
}

\section{Cómo citar / How to cite}

Zapata-Aguilar, J. A., Pérez-Akaki, P., \& Moo-Novelo, C. A. (2020). Análisis de la cadena de comercialización del chile habanero de Yucatán y su denominación de origen. Revista CEA, 6(11), 109-125. https://doi.org/10.22430/24223182.1406

Recibido: 29 de junio de 2019

Aceptado: 3 de octubre de 2019

\section{Resumen}

Las cadenas de comercialización se han vuelto un instrumento importante para el estudio de las relaciones económicas, pues permiten entender los diferentes procesos, agentes y características de un bien, desde su origen hasta que llega a manos de los consumidores. El presente estudio busca caracterizar la cadena de comercialización del chile habanero de Yucatán a través de una investigación documental y de campo, para conocer su funcionamiento y el impacto que ha generado sobre dicha cadena la obtención de la denominación de origen-DO-. Se emplearon dos técnicas de recolección de información: la primera, fue una investigación documental y la segunda fue una entrevista semiestructurada dirigida a actores de la cadena de comercialización del chile habanero. Se encontró como resultado del estudio de investigación, de enfoque cualitativo, que los

* Este artículo se deriva del proyecto titulado «Análisis de la cadena de comercialización del chile habanero de Yucatán y su denominación de origen» y ha sido financiado con recursos propios. 
intermediarios son los actores más beneficiados de la cadena, y no los productores como se podría pensar; así mismo, se halló que la denominación de origen no está siendo aprovechada como se planeó, ya que, al no cumplirse con la normatividad que exige la DO, no es posible usar este distintivo para obtener un sobreprecio por esta importante hortaliza en los mercados internacionales. Por tanto, resulta indispensable la creación de un Consejo Regulador del Chile Habanero que siente las bases para la integración de la cadena, que en este caso deberá ser peninsular ya que la DO contempla también a los estados de Campeche y Quintana Roo.

Palabras clave: abastecimiento, comercio, mercado, cadena de valor, denominación de origen.

Clasificación JEL: K10, F14, M38.

\begin{abstract}
Supply chains have become important tools to study economic relationships because they can be used to understand different processes, agents, and characteristics involved in a product, from its origin until it reaches consumers' hands. This study aims to characterize the supply chain of Yucatán habanero pepper, adopting a documentary method and field research, in order to examine its operation and the impact it has experienced since the Protected Designation of Origin (PDO) was obtained. Two techniques were employed to collect information: (1) documentary research and (2) semi-structured interviews with the actors in the supply chain of habanero pepper. This qualitative study found that intermediaries (not producers, as one might think) benefit the most from the supply chain. Furthermore, the PDO is not being exploited as planned because, as the standards required for the PDO are not met, the logo cannot be used to obtain a surcharge for this important vegetable in international markets. For that reason, a regulatory commission for habanero pepper should be created to establish the foundations to integrate its supply chain, which would cover the Yucatán peninsula because the PDO also includes the States of Campeche and Quintana Roo.
\end{abstract}

Keywords: Supply, commerce, market, supply chain, designation of origin.

JEL classification: K10, F14, M38.

\title{
1. INTRODUCCIÓN
}

De acuerdo con González Andrade (2015) y Fearne et al., (2012), el análisis de la cadena de valor es importante porque las ventajas competitivas se crean a partir de la diferenciación del producto y de la reducción de costos. Para lograr obtener dichas ventajas es necesario el uso de modernas tecnologías, la innovación y los factores especializados (Mudambi \& Puck, 2016). Así, la presente investigación busca conocer los efectos que a la fecha ha generado la denominación de origen (DO) del chile habanero en su cadena de comercialización, ya que originalmente se pensó que esta sería el detonador del cultivo de este producto en la península de Yucatán, y no ha resultado así. El problema es que no se tiene un conocimiento público de la estructura actual de la cadena de comercialización de este fruto y, por lo tanto, no se puede proyectar su integración, contemplando a los diferentes actores que la conforman. 
El estudio contempló una investigación documental y se empleó un diseño no experimental transversal, abordando a los diferentes actores de la cadena de comercialización del chile habanero a través de entrevistas semiestructuradas dirigidas a: productores, comercializadores, empresarios industriales y representantes del Gobierno, vinculados con la compra-venta de chile habanero.

El artículo está conformado de seis apartados, siendo el primero el marco teórico, en donde se plasman los resultados de la investigación documental realizada, que engloba las aportaciones de diferentes autores respecto al tema abordado en la investigación. El segundo apartado contempla la metodología empleada que incluye la manera como se realizó la investigación, las técnicas de recolección de información usadas, el instrumento empleado en la recopilación de datos, así como el proceso paso a paso que se siguió para efectuar la investigación. El tercer apartado presenta los principales hallazgos encontrados en la investigación, el cuarto incluye la discusión derivada de los resultados obtenidos, el quinto contempla las ideas finales destacables de los hallazgos encontrados en el estudio de investigación y el sexto apartado presenta una serie de recomendaciones a considerar en la operación de la cadena de comercialización del chile habanero, en busca de aprovechar la DO.

\section{MARCO TEÓRICO}

\section{Importancia de las cadenas de valor para el desarrollo rural}

Para la Comisión Económica para América Latina y el Caribe (CEPAL) (2016) una cadena de valor

comprende toda la variedad de actividades que se requieren para que un producto o servicio transite a través de las diferentes etapas de producción, desde su concepción hasta su entrega a los consumidores y la disposición final después de su uso (parr. 1).

De acuerdo con García Muñiz y Solís Arias (2014), es importante medir el valor añadido, es decir, quién y dónde se crea el valor de lo que se intercambia. Esto como elemento fundamental del funcionamiento de la cadena de valor, ya que permite verificar que todos los participantes están realizando su aportación al sistema (Biggeri et al., 2017).

Por otro lado, de acuerdo con Bianchi y Szpak (2013, p. 7), el surgimiento de los sistemas internacionales de producción integrada o redes globales de producción, denominados frecuentemente como cadenas de producción o cadenas globales de valor, están asociados a la llamada «segunda desagregación» de la globalización, caracterizada por los rápidos avances de las tecnologías de la información y las comunicaciones, los menores costos de transporte, la liberalización del comercio y el auge de la inversión extranjera directa. Estos factores han hecho rentable la combinación de recursos de diferentes países a fin de producir un bien (Los et al., 2015).

Del mismo modo, como menciona Barrientos Felipa (2015, p. 3) así como Kuijpers y Swinnen (2016), para desarrollar cualquier estrategia de comercialización de productos es importante considerar que la producción local se basa en el esfuerzo de pequeños agricultores, que se convierten en parte importante de una cadena de valor. Otro elemento que, de acuerdo con Tamayo Manrique et al., (2014), podría potencializar las cadenas de valor, es la producción agrícola libre de químicos, que es actualmente una actividad importante dentro del comercio mundial $y$, debido a su constante 
crecimiento, puede llegar a constituir en mediano y largo plazo una fuente de ingresos para las familias de los pequeños y medianos productores, así como una fuente de empleo para el área rural (p. 6).

Para Kosacoff et al., (2007, p. 50) y Stevens (2001), una mayor participación de las empresas locales en las cadenas de valor nacionales, regionales y globales, hace posible aumentar la productividad, fortaleciendo la competitividad, al enfrentarse a demandas cada vez más exigentes, estimulando el desarrollo de nuevos procesos de aprendizaje que derivan de las relaciones establecidas con otros actores de la cadena, contribuyendo significativamente a la mejoría de la condiciones, económicas y sociales del entorno. Para ello se desarrolló una investigación documental, así como un levantamiento de información primaria por medio de visitas de campo, usando las dimensiones y variables acordes con la teoría de las Cadenas Globales de Mercancías (CGM) (ver Tabla 1).

Tabla 1. Dimensiones y variables consideradas en el estudio de la cadena productiva del chile habanero

Table 1. Dimensions and variables in the study of the supply chain of habanero pepper

\begin{tabular}{ll}
\hline \multicolumn{1}{c}{ Dimensión } & \multicolumn{1}{c}{ Variables } \\
\hline Entradas y salidas & $\begin{array}{l}\text { Características del producto } \\
\text { Estructura de la cadena } \\
\text { El mercado }\end{array}$ \\
\hline Cobertura geográfica & Geografía de la producción \\
\hline Gobernanza & Control de la cadena \\
\hline Marco institucional & Normatividad \\
\hline
\end{tabular}

Fuente: elaboración propia.

\section{Las denominaciones de origen y su importancia en el reordenamiento de las cadenas de comercialización}

La vinculación de un producto con una región determinada, así como la facultad de impedir su uso cuando no esté autorizado, otorga a la DO la característica de signo distintivo que permite su identificación y reconocimiento, motivo por el cual se incluye en materia de derecho de la propiedad industrial (Molina, 2015). Sin embargo, algunos han considerado que, debido a su doble papel como identificador del origen del producto e indicador de su calidad, pueden considerarse, tanto signos distintivos de la propiedad industrial como figuras jurídicas independientes de dicha institución. El lugar de origen puede ser considerado una fuente valiosa de ventajas únicas para las empresas, en tanto es susceptible de influir sobre la valoración que los consumidores finales les dan a los productos (Trejo-Pech et al., 2010). Resulta relevante destacar el papel desempeñado por la imagen de un producto o marca en el proceso previo a la compra desarrollada por el consumidor y, al mismo tiempo, el lugar de fabricación o procedencia repercute en la representación mental que sobre el producto o marca se crea el consumidor (Martín Cerdeño, 2006, p. 94). Según Hernández Rojas (2018):

El incremento de la demanda de productos diferenciados tanto en materia prima como a través de platos, que llevan implícitas, materias primas diferenciadas, ha propiciado el desarrollo de marcas o insignias específicas para las materias primas, en concreto las Denominaciones de Origen Protegidas (DOP) e Indicaciones Geográficas Protegidas (IGP) (p.3). 
De acuerdo con Granados Rojas (2004), la diferenciación del producto, además de constituir una oferta diferente y ser atractiva para el consumidor, debe cumplir otras características:

a. "Ofrecer un valor superior al cliente frente a los productos similares.

b. Su base diferenciadora debe ser sostenible y defendible a largo plazo, es decir, no debe poder ser imitada con facilidad.

c. Debe ser económicamente viable» (p. 15).

Molano Pineda et al., (2017) señalan que «Las DO se encargan de certificar la calidad y procedencia de un producto cuyo método de producción y elaboración está culturalmente arraigado en una región» (p.194). Para organismos como la Organización Mundial de la Propiedad Intelectual (OMPI) el objetivo de una DO es mejorar la posición en el mercado de un producto, a través de la estandarización de la calidad, la protección de los plagios de la competencia y, por consiguiente, de su reputación. Sin embargo, hay otros beneficios no menos importantes que no se deben perder de vista, como el rescate y la protección de los recursos internos, como son las materias primas, las técnicas de elaboración, la mano de obra y la gestión con los actores locales, que permitan no solo su implementación, sino el control de la DO y el reforzamiento de los lazos de identidad regional, en beneficio de toda la cadena productiva, pues pueden promover alianzas y asociaciones de empresas pequeñas y medianas, así como exportar las distintas especialidades, generando riqueza al país y a las respectivas economías regionales (Errázuriz Tortorelli, 2010, p. 211).

Por otro lado, las DO dan garantías a los consumidores respecto a la calidad del bien. En el momento en que el público evalúa los productos agroalimentarios con este tipo de reconocimientos, identifica su calidad en atributos, tanto intrínsecos, que identifican al producto -tales como los métodos de elaboración, controles de calidad y trazabilidad, los cuales se ven reflejados en las propiedades físicas del mismo como el color, el olor, la apariencia y el sabor-, así como los atributos extrínsecos, los cuales se refieren a la calidad percibida por el consumidor respecto a la marca -el empaquetado, etiquetado, precio, la región de origen, entre otros-. Esta doble dimensión le permite al consumidor evaluar y tomar una decisión de compra hacia este tipo de productos (Espejel Blanco \& Fandos Herrera, 2009, p. 146). Incluso, el cumplimiento de esos estándares, permite que los consumidores estén dispuestos a pagar más por ellos (Valenciano \& Román Sánchez, 2011, p. 136).

De acuerdo con Cava Jiménez et al., (2019), «los productos agrícolas que cuentan con una DOP (denominación de origen protegida), IGP o TSG son sinónimo de calidad y gran demanda» (p.5). Por tanto, este reconocimiento debería también representar un cambio en el control de mando de la cadena -dimensión gobernanza- a favor de los productores, permitiéndoles obtener mayores beneficios sobre su bien, así como controlar y tener una mayor participación en la determinación del rumbo futuro de dicho bien agrícola.

\section{El chile habanero}

Este fruto es una baya hueca en forma de trompo, poco carnosa, con dos y hasta ocho hojas modificadas, que constituyen el aparato reproductor femenino de la flor y se denominan carpelos. El chile habanero ocupa un lugar principal entre los productos agrícolas de Yucatán. Tradicionalmente se vende fresco para su consumo directo o como una materia prima para preparar salsas picantes, pero también existen diferentes empresas en el mercado que se encargan de procesarlo y 
distribuirlo, tanto en el país como en el extranjero, en forma de chile seco entero, en polvo, pasta y en hojuelas (Maldonado Reyes, 2013).

Según Ocampo Thomason et al., (2014), del 2002 al 2012 la producción de chile habanero a nivel nacional se concentró en 17 entidades federativas; no obstante, los dos estados que sobresalen fueron Yucatán y Tabasco (p. 19). Por su parte, los estados de Campeche y Quintana Roo presentan una producción de chile habanero creciente en los últimos años, aunque relativamente menor a la generada por los dos estados líderes; sin embargo, podrán beneficiarse de la DO del chile habanero, por localizarse en la península de Yucatán.

\section{METODOLOGÍA}

La presente investigación se realizó en tres partes: en la primera parte se definió el diseño a utilizar, que en este caso fue no experimental transversal, debido a que los participantes en el trabajo de campo solo se abordaron en una ocasión, es decir, no se realizó un segundo levantamiento para verificar los resultados obtenidos en primera instancia, razón por la cual se considera no experimental transversal. En esta primera parte, se seleccionaron dos técnicas de recolección de información: la primera fue la investigación documental, con la que se pudo obtener información sobre los volúmenes producidos de chile habanero en años anteriores, así como los principales estados que se dedican a esta actividad, destacando también el tipo de tecnología empleada en cada unidad de producción, así como el tamaño de las unidades agrícolas, con lo cual se pudo caracterizar en primera instancia la actividad.

Posteriormente, en la segunda fase del estudio se aplicó la entrevista semiestructurada de enfoque cualitativo, que se dirigió a productores, comercializadores, empresarios industriales y representantes del Gobierno relacionados con la producción de chile habanero en la península de Yucatán. Se entrevistaron veintidós productores localizados en la zona sur del estado de Yucatán, de los municipios de Tekax, Yaxcabá, Tixmeuac, Timucuy y Halacho, dos intermediarios, dos empresarios industriales, un representante de los productores de chile habanero y un funcionario público vinculado con su cultivo. La muestra fue no aleatoria por conveniencia, ya que respondió a dos criterios: el primero fue que los entrevistados participaran activamente en la cadena de comercialización del chile habanero y la segunda su disposición para participar en el estudio de investigación.

En la segunda parte del estudio se realizó toda la investigación documental, tomando como referencia las dimensiones y variables para la valoración de la cadena de comercialización del chile habanero de la península de Yucatán, de acuerdo con la propuesta metodológica de Gereffi (2001).

En la tercera parte se entrevistaron treinta participantes que incluyeron productores, comercializadores, empresarios industriales y representantes del Gobierno relacionados con la producción de chile habanero en la península de Yucatán, para conocer la manera cómo funciona la cadena, tomando como eje la investigación documental previamente realizada. A partir de la información recolectada se realizó un análisis a profundidad de las respuestas proporcionadas por los participantes, las cuales se cruzaron en busca de puntos de coincidencia, para confirmar o en su caso ajustar la cadena de comercialización del chile habanero hallada en la investigación documental 
y caracterizarla, así como valorar el impacto que la denominación de origen ha tenido sobre ella. El estudio inició en el mes de agosto del año 2014, para concluir en el mes de febrero de 2018.

\section{RESULTADOS}

\section{Características del producto}

El chile habanero en la península de Yucatán es como el vino en Francia, se trata de un bien ampliamente consumido por la población, pero que acompaña prácticamente a toda la gastronomía de la región. Es tan común esta hortaliza en la dieta que regularmente los habitantes cuentan con plantas de habanero en sus patios traseros, lo que les ofrece la posibilidad de consumirlo diariamente. Se encontró en la investigación tres clases de chile habanero, el de primera que se caracteriza por su tamaño y atractiva apariencia, el de segunda que es más pequeño, pero de buen aspecto, y el de tercera que además de ser pequeño suele ser visualmente poco atractivo.

\section{La cadena de comercialización del chile habanero de Yucatán}

A partir de la observación directa del trabajo de campo, se identificó que la cadena de comercialización del chile habanero se clasifica en cuatro niveles (ver Figura 1). En el primer eslabón se ubican los productores rurales que, de acuerdo con el Plan Rector del Sistema Producto Chile Habanero de Yucatán, se pueden clasificar en tres tipos: los pequeños parcelarios que hacen siembras de traspatio, mejor conocidos como mecateros -haciendo referencia a un mecate, que es un área de cultivo de 400 metros cuadrados-, los cuales se consideran en el perfil de los productores de baja tecnología; los que cultivan menos de 15 hectáreas, son quienes figuran como productores de tecnología media; y recientemente algunos particulares que se están incorporando a la actividad agrícola con una producción mayor a las 15 hectáreas, que se consideran de tecnología media-alta (Secretaría de Agricultura, Ganadería, Desarrollo Rural, Pesca y Alimentación [SAGARPA], 2012, p. 5).

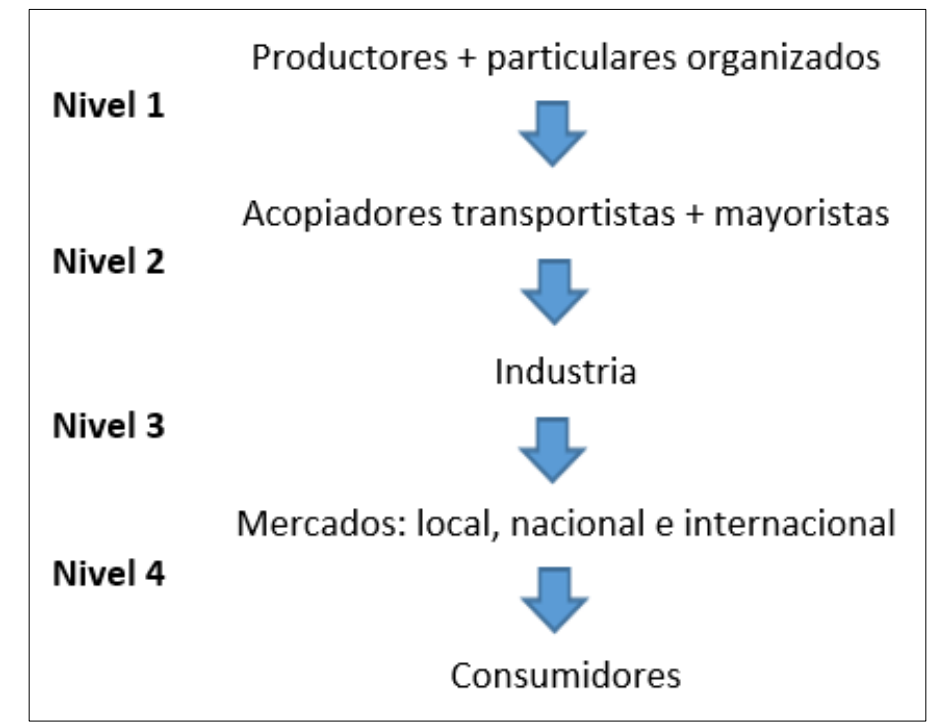

Figura 1. Cadena de comercialización del chile habanero

Figure 1. Supply chain of habanero pepper

Fuente: elaboración propia. 
Los productores de tecnología media y baja se encuentran dispersos en localidades y comisarías de los municipios de la península de Yucatán, a distancias de uno a seis kilómetros a la redonda de la cabecera municipal. Las tierras que trabajan son principalmente ejidales, compran sus semillas a través de las empresas agropecuarias localizadas en el centro de los municipios en los que habitan o bien preparan sus semilleros y de ahí obtienen las plántulas para la siembra, pues son cultivos de temporal principalmente. Por su parte, los particulares organizados son productores nacionales e incluso extranjeros, con formación académica y experiencia en el cultivo de hortalizas cíclicas, que realizan una función controlada y sus cosechas son dirigidas principalmente a los mercados nacionales e internacionales.

En un segundo nivel se encuentran los acopiadores, intermediarios transportistas o mayoristas, quienes son normalmente comerciantes de las principales cabeceras municipales que cuentan con vehículo propio y suficiente liquidez. Estos agentes compran el chile disponible a nivel de parcela, estableciendo el precio de compra, generalmente muy por debajo del precio de mercado, dejando en desventaja al productor, que no tiene forma de llevar su producto para venderlo a los mercados locales. Si el productor se negara a vender a los intermediarios y decidiera ir a los mercados a ofertarlo directamente, es muy probable que sea el intermediario transportista o mayorista quien lo adquiera, incluso a un menor precio, como castigo para el productor por negarse a venderlo en la parcela, lo que desalienta a los productores a tratar de saltar al comprador local de chiles. Posteriormente, estos intermediarios mayoristas y transportistas trasladan el chile habanero en fresco y las otras hortalizas adquiridas en su recorrido a los principales mercados de consumo local y nacional, o bien a los detallistas e industriales. El chile habanero de mayor calidad se comercializa en fresco, ya que por sus características es demandado por los diferentes mercados.

En el tercer nivel se localizan los industriales, quienes compran el chile habanero de segunda fresco a los intermediarios mayoristas, denominado así por su apariencia, aun cuando el nivel de picor sea igual o mayor al de un chile habanero de primera. Es adquirido para ser procesado y convertirlo en pasta, que es usada como materia prima en la elaboración de salsas que se venden a los detallistas como los supermercados, restaurantes, entre otros. Parte de la producción de chile habanero en pasta es vendida como materia prima a industrias alimenticias pequeñas y la restante se reserva como insumo para seguir elaborando salsas y condimentos de chile. En Yucatán se identifican dos tipos de industrias que se dedican al procesamiento del chile habanero: por su tamaño, las primeras son las industrias medianas que tienen entre 51 y 250 empleados, entre las que destacan Condimentos y Salsas La Anita S.A. de C.V., Salsas y Condimentos El Yucateco S.A. de C.V. y Productos La Extra; el segundo tipo está integrado por las microindustrias con menos de diez empleados, entre las que destacan: Fuego Maya S.P.R. de R.L., Alimentos y Aderezos del Sureste S.A. de C.V., entre otros. No se encontró evidencia de la existencia de formas organizativas entre los integrantes de estas industrias.

El cuarto nivel lo ocupan los comercializadores en los mercados local, nacional, internacional y los detallistas. En cuanto a la vía local y nacional, los principales mercados en Yucatán son la Central de Abastos y la Casa del Pueblo en Mérida, así como la Central de Abastos de Oxkutzcab. A nivel nacional los principales mercados son la Central de Abastos de Iztapalapa en el D.F., el Mercado de Abasto de Monterrey y la Central de Abasto INDIA de Tijuana, cuya cercanía con California -donde habitan miles de connacionales- permite llevar el chile habanero al «mercado de la nostalgia». Con respecto al mercado internacional, los intermediarios mayoristas y los particulares organizados venden el 
producto en fresco a comisionistas especializados (brokers), quienes se encargan de llevar el producto hacia mercados en Los Ángeles, Miami, Nueva York, San Antonio y Houston.

Se detectó en el trabajo de campo realizado que los productores de tecnología media y baja conocen parcialmente los estándares de calidad requeridos para ostentar la DO en el cultivo del chile habanero, aun cuando hacen uso de semillas criollas, las cuales no son en todos los casos semillas certificadas. Además se evidenció que, a pesar de que los cultivos son vigilados por Sistema Nacional de Sanidad, Inocuidad y Calidad Agroalimentaria (SENASICA) y constantemente los productores están siendo instruidos en la sustitución de agroquímicos dañinos para los cultivos por otros amigables con el medio ambiente, algunos los siguen usando por considerar que los bionaturales no son tan efectivos como los primeros, existiendo la posibilidad de cosechar productos bajo el efecto de agroquímicos prohibidos que frenarían el ingreso del chile habanero a los mercados internacionales.

Por otro lado, una DO requiere la instalación de un consejo regulador, lo que no ha podido lograrse entre los actores del sector. El liderazgo del representante del Consejo Estatal de Productores de Chile Habanero (CEPROCH), quien con su empuje llegó a ser también el presidente del Sistema Producto Chile de Yucatán, permitió tener un mayor acercamiento entre los diferentes actores, tanto directos como indirectos, gestando un diálogo entre ellos a favor de un mejor escenario para la constitución del consejo regulador antes mencionado.

La participación de actores externos a la cadena -entre los que pueden citarse centros de investigación (CICY, INIFAP), proveedores de insumos (Agropecuaria del campesino) y universidades (ITM, Universidad Tecnológica del Mayab, FCA de la UADY) - ha generado una dinámica a favor en temas relacionados con la hortaliza, que incluyen actividades de investigación, capacitación y gestión del conocimiento.

Los programas agropecuarios de impulso a través de dependencias federales como SAGARPA, y estatales como la Secretaría de Desarrollo Rural (SEDER), no están siendo oportunamente aprovechados por los productores agrícolas ante una falta de organización y una débil capacidad de gestión.

Con respecto a la denominación de origen se encontró que, aunque los participantes de la cadena de comercialización del chile habanero entrevistados en la investigación están enterados de que la hortaliza cuenta con este distintivo, también saben que no pueden hacer uso de él, en tanto no exista un consejo regulador que se encargue de su administración; por lo tanto, no se está aprovechando como se hubiera deseado.

En el análisis de la gobernanza de la cadena del chile habanero se discute sobre la forma en la que se organizan los actores y los aspectos normativos que se utilizan para garantizar el flujo del producto, desde el productor hasta los consumidores. Por ello, las dimensiones a estudiar aquí son la estructura de los mercados, la distribución de ganancias en la cadena, el marco institucional, las posibilidades de mejoramiento de su posición y, finalmente, el análisis de control de mando. 


\section{Estructura de los mercados de producción y comercialización}

El Estado de Yucatán cuenta con 106 municipios, de los cuales la capital es la ciudad de Mérida. Su superficie representa el $2.02 \%$ del total nacional, el $85 \%$ de la superficie presenta clima cálido subhúmedo y el $14.5 \%$ presenta clima seco y semiseco, que se localiza en la parte norte del estado. Tiene una población de 2'097.175 habitantes, de los cuales el $84 \%$ vive en zonas urbanas y el $16 \%$ en zonas rurales, precisamente en la zona de clima cálido subhúmedo en donde se desarrolla el cultivo del chile habanero, el presidente del Consejo Estatal de Productores de Chile Habanero, (A. Ríos, comunicación personal, 26 de septiembre de 2014), señaló que se tienen identificados 1.532 productores en el estado de Yucatán. Es preciso comentar que dichos productores también desarrollan otros cultivos cíclicos y perennes, y que se encuentran localizados en los municipios de Muna, Yaxcabá, Peto, Mérida, Halachó, Timucuy, Santa Elena, Tekax, entre otros, concentrados principalmente en tres regiones: tres municipios productores se ubican en la región centro, siete en la región sur y tres más se encuentran ubicados en la región oriente. Esta situación generó, de acuerdo al Servicio de Información Agroalimentaria y Pesquera, que Yucatán fuera el primer productor a nivel nacional, con un total de 26.555 toneladas (ver Figura 2).

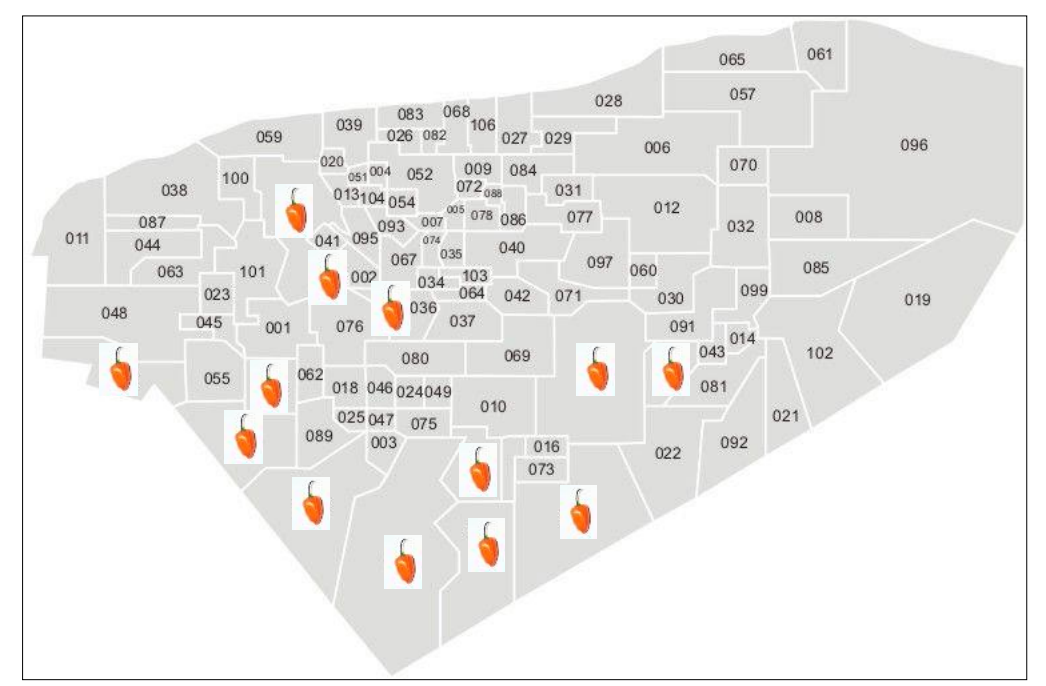

Figura 2. Municipios productores de chile habanero en el estado de Yucatán

Figure 2. Municipalities that produce habanero pepper in the State of Yucatán Fuente: elaboración propia a partir de Ríos (2014).

\section{DISCUSIÓN}

Según Flores Novelo et al., (2008) «el canal del chile habanero muestra un claro modelo de oferta y demanda dispersas» (p. 11). Este argumento se confirma ya que, como puede observarse en la Figura 2 , existe una alta dispersión de los productores en el estado, lo que dificulta su integración y coordinación para el aprovechamiento de recursos en concurrencia. Esta condición no favorece a los productores, pues encarece los costos de traslado del bien hacia los mercados de consumo, además que los vuelve vulnerables a la participación de agentes acopiadores del producto.

De acuerdo con uno de los productores de chile habanero entrevistados de la localidad de Cepeda en el municipio de Tekax, Yucatán, debido a que varios de los insumos empleados en los cultivos, 
como son insecticidas y fertilizantes sintéticos, son de importación, cuando el precio del dólar sube, el precio de los agroquímicos se eleva hasta en un $20 \%$, resultando en ocasiones incosteables.

\section{Distribución de las ganancias a lo largo de la cadena del chile habanero}

Los agricultores venden su producto a los intermediarios transportistas, quienes conocen a detalle los períodos de cosecha y las rutas de contacto, y han establecido previamente comunicación con los productores desde la siembra, facilitando la compra del chile habanero. El intermediario transportista o acopiador suele vender también al intermediario mayorista. Por su parte, el intermediario mayorista puede comprar directamente a los productores o al intermediario transportista, para atender su mercado, conformado por supermercados, industrias, restaurantes, entre otras empresas, teniendo como ventaja el hecho que les puede facturar. El intermediario transportista cuenta con los recursos económicos suficientes para pagar los gastos de transporte del producto, llevarlo a los centros de abasto y clasificarlo para ser canalizado a los mercados locales y nacionales. Los productores e intermediarios observan la calidad del producto en términos de su aspecto físico como: tamaño, color, aroma y picor, sin tener claros los estándares de calidad exigidos por los mercados internacionales, ni mucho menos los estipulados en la NOM para la DO, debido a que tanto el mercado local como el nacional no les exige más. Este hecho sin duda tiene que ser considerado, ya que, sin el cumplimiento de la norma, no se podrá aprovechar la DO.

Se observó que el intermediario es el más beneficiado debido a que el agricultor es el que corre con todos los riesgos de producción, desde la siembra hasta la cosecha, mientras que el intermediario invierte en el transporte y entrega. El detallista, por su parte, es el que más recibe por cada peso vendido, pero se hace responsable de su conservación y mantenimiento, corriendo el riesgo de que si no logra venderlo debe desecharlo por tratarse de un producto perecedero.

Los elevados costos de producción de la hortaliza vuelven vulnerables a los productores, dadas las variaciones del precio del chile habanero en el mercado, en virtud de que las plantas de este bien agrícola requieren fertilización y fumigación constante para combatir las múltiples plagas que la afectan a lo largo de su ciclo de vida. Según uno de los agricultores entrevistados del municipio de Tixmehuac, los costos de producción del chile habanero representan entre un $40 \%$ y un $80 \%$ del precio de venta de la hortaliza, por lo que es a partir de la tercera cosecha que se empieza a obtener ganancias por su venta.

La distribución desigual de la riqueza en la cadena, que se concentra en los intermediarios y los industriales, deja en desventaja a los productores, quienes tienen que enfrentar los costos galopantes que exige la conservación de los cultivos, propiciando esto que algunos agricultores hayan sustituido al chile habanero por otros productos del campo, con mayor demanda y menor costo de producción.

Un elemento importante para lograr las máximas ganancias en las actividades económicas es incrementar la productividad y, por lo tanto, la competitividad. Por esta razón es importante considerar, de acuerdo con Smith en la Riqueza de las Naciones (1776), citado por Moo Xix et al., (2016), que un país debería especializarse en aquellas actividades que maximizaran su beneficio -la vocación del país-, exportando productos donde se tuviera una ventaja absoluta (p. 2), con lo que se podría obtener los beneficios que se esperan de la actividad económica, lo cual estaría ligado también con las cadenas de valor que se generan. 


\section{Marco institucional internacional, nacional y regional de la cadena de chile habanero}

La Asociación Civil Chile Habanero de Yucatán conformado por productores, comercializadores e industriales, inició la gestión de la DO en el mes de agosto del año 2005. Posteriormente, en el mes de noviembre de 2007, los estados de Campeche y Quintana Roo solicitaron su adhesión, misma que les fue rechazada inicialmente. Por medio de negociaciones a nivel de gobernadores de las entidades, consiguieron su inclusión, lo que quedó plasmado en la declaratoria publicada en el Diario Oficial de la Federación (DOF) el 4 de junio de 2010. El 9 de noviembre de 2011, el Chile Habanero de la Península de Yucatán obtuvo su registro internacional con el número 898 ante la Organización Mundial de la Propiedad Industrial (OMPI).

Dos años más tarde, el 15 de junio de 2012, se publicó en el Diario Oficial de la Federación (tercera sección, parr.1), el proyecto de norma oficial mexicana PROY-NOM-189-SFCl-2012 Chile Habanero de la Península de Yucatán (Capsicum Chinense Jacq), con la finalidad de someterlo a la opinión de expertos antes de su declaración. El proyecto de norma incluye especificaciones y métodos de prueba a los que deberán someter el producto. La norma fue aprobada el 28 de septiembre de 2012, en la tercera sesión ordinaria del comité consultivo nacional de normalización de seguridad al usuario, información comercial y prácticas de comercio, y publicada en el DOF el 30 de noviembre de 2012.

La DO protege al chile habanero en las modalidades fresco, en estado inmaduro o maduro, encurtido, en pasta, deshidratado entero y en polvo, así como en salsas. La NOM-189-SCFI-2012 contempla en su estructura las siguientes normas mexicanas (ver Tabla 2).

Tabla 2. Normas oficiales mexicanas contempladas en la NOM-189-SCFI-2012

Table 2. Official Mexican regulations in NOM-189-SCFI-2012

\begin{tabular}{|c|c|}
\hline Norma & Descripción \\
\hline NOM-051-SCFI/SSA1-2010 & $\begin{array}{l}\text { Especificaciones generales de etiquetado para alimentos y bebidas no } \\
\text { alcohólicas pre envasados. Información comercial y sanitaria publicada } \\
\text { en el Diario Oficial de la Federación el } 5 \text { de abril de } 2010\end{array}$ \\
\hline NOM-092-SSA1-1994 & $\begin{array}{l}\text { Bienes y servicios. Método para la cuenta de bacterias aerobias en placa } \\
\text { publicada en el Diario Oficial de la Federación el } 12 \text { de diciembre de } 1995\end{array}$ \\
\hline NOM-106-SCFI-2000 & $\begin{array}{l}\text { Características de diseño y condiciones de uso de la contraseña oficial, } \\
\text { publicado en el Diario Oficial de la Federación el } 2 \text { de febrero de } 2001\end{array}$ \\
\hline NOM-111-SSA1-1994 & $\begin{array}{l}\text { Bienes y servicios. Método par cuenta de mohos y levaduras en } \\
\text { alimentos, publicada en el Diario Oficial de la Federación el } 13 \text { de } \\
\text { septiembre de } 1995 \text {. }\end{array}$ \\
\hline NOM-113-SSA1-1994 & $\begin{array}{l}\text { Bienes y servicios. Método para la cuenta de microorganismos } \\
\text { coliformes totales, en placa, publicada en el Diario Oficial de la } \\
\text { Federación el } 25 \text { de agosto de } 1995\end{array}$ \\
\hline NOM-115-SSA1-1994 & $\begin{array}{l}\text { Bienes y servicios. Métodos para la determinación de Stophylococcus } \\
\text { Aureus, en alimentos, publicada en el Diario Oficial de la Federación el } 25 \\
\text { de septiembre de } 1995\end{array}$ \\
\hline NOM-251-SSA1-2009 & $\begin{array}{l}\text { Prácticas de higiene para el proceso de alimentos, bebidas o } \\
\text { suplementos alimenticios, publicada en el Diario Oficial de la Federación } \\
\text { el } 1 \text { de marzo de } 2010\end{array}$ \\
\hline
\end{tabular}


La norma que regula el chile habanero contempla la clasificación del producto, los reconocimientos o certificaciones que debe tener el área de producción y empaque en materia de reducción de riesgos, los lineamientos que establece la Secretaría de Salud en el implemento de aditivos y coadyuvantes en alimentos, las especificaciones en materia de muestreo y métodos de prueba, los órganos de vigilancia, así como también las normas internacionales consultadas en la elaboración del proyecto de norma para el chile habanero.

\section{Posibilidades de mejoramiento en la cadena}

La cadena de comercialización del chile habanero presenta desorganización entre sus integrantes, y falta de compromiso compartido enfocado al trabajo en equipo, en el que todos los eslabones de la cadena salgan beneficiados, desde la tarea que les corresponda realizar. El actual líder del CEPROCH se esfuerza por vincular a los diferentes eslabones, sin embargo, la integración requiere recursos económicos constantes, que actualmente están actuando en sentido contrario a los esfuerzos que se vienen haciendo por conseguir la constitución del Consejo Regulador del Chile Habanero, que en este caso tendrá que ser peninsular, en virtud de que la DO también contempla a los estados de Campeche y Quintana Roo.

Este consejo regulador peninsular podría incrementar las posibilidades de mejoramiento de la cadena de comercialización, ya que entre sus funciones tendría la definición de las responsabilidades de cada eslabón y la supervisión del cumplimiento de los compromisos acordados, además de que sentaría las bases para la consolidación de la comercialización del producto agrícola a nivel regional, nacional e internacional, de acuerdo a la normatividad exigida por la DO. Esto podría llevar a aprovechar mejor los nichos de mercado prometedores, como el europeo y el asiático, considerando que el principal mercado, que es los Estados Unidos, presenta a partir del año 2017 un escenario incierto. Los industriales y los intermediarios tienen claro los beneficios que representa la denominación de origen, como distintivo para cobrar un sobreprecio por el chile habanero en fresco, no obstante, los productores, figuras eje del proceso, aun cuando han identificado lo anteriormente expuesto, no logran concretar la inocuidad en el cultivo de la hortaliza, para dar cumplimiento a la normatividad que exige la denominación de origen.

\section{Control de mando de la cadena de comercialización de chile habanero de la península de Yucatán}

Los intermediarios o comercializadores tienen el control de la cadena debido a que son el eslabón de enlace entre los productores que, dada su dispersión y falta de organización, terminan trabajando para el intermediario, los mayoristas y detallistas, quienes colocan el producto en manos del consumidor una vez que el intermediario les surte la hortaliza. La principal ventaja competitiva del intermediario es su conocimiento sobre las tendencias de la demanda del mercado, preparándose con anticipación para los períodos de escasez y comprando a bajo precio en los períodos de sobre oferta.

\section{CONCLUSIONES}

Las cadenas de comercialización son un valioso mecanismo para vincular los esfuerzos de los diferentes actores que la conforman, tanto de los actores directos que son los que producen, 
intermedian y transforman el bien agrícola, en este caso el chile habanero, como de los indirectos, que, con sus aportes científicos, técnicos y operativos, facilitan el cultivo y comercialización de la hortaliza. No obstante, todo el trabajo anterior no será suficiente sin la oportuna articulación de los actores involucrados y su compromiso para coadyuvar al dinamismo de esta importante cadena.

Se encontró que la cadena de comercialización del chile habanero de la península de Yucatán no la dirige el productor, sino el comprador, específicamente los intermediarios, los mayoristas y detallistas, por lo que la distribución actual de la riqueza deja en desventaja al productor de tecnología media y baja, quien asume la mayor responsabilidad en el cultivo de la hortaliza que ya de entrada le resulta cara, no solamente por lo que implica su cultivo, sino porque requiere vigilancia permanente contra plagas, y ser atendida sustituyendo plaguicidas por productos amigables con el medio ambiente que permitan cumplir con los estándares exigidos en los mercados internacionales, si su destino fuera la exportación.

La dinámica antes expuesta está llevando a los productores de chile habanero a sembrar otros productos agrícolas para amortiguar los costos del cultivo y están dejando de sembrarlo al descubrir que otros frutos les generan mejores ingresos y menores costos de producción.

Los intermediarios, mayoristas y detallistas, gobiernan por el momento la cadena de comercialización del chile habanero, ya que, sin arriesgar capital en los cultivos, ni enfrentar las múltiples dificultades que implica sembrar la hortaliza, compran a precios accesibles para vender a diferentes mercados con la ventaja de poder facturarles, requisito indispensable entre sus clientes y ofreciéndoles crédito en algunos casos.

Con respecto a la denominación de origen, esta no está siendo aprovechada en la comercialización del chile habanero de la península de Yucatán. La DO reclama el cumplimiento de toda una normatividad $y$, desafortunadamente, la mayor parte de los productores de chile habanero de la península de Yucatán no la conocen completamente, lo que los frena a cultivar la hortaliza de acuerdo con las especificaciones que reclama la norma y cosechar un producto calificado para ostentar el distintivo.

Resulta urgente la creación de un Consejo Regulador Peninsular para la Denominación de Origen del Chile Habanero, que siente las bases para el cumplimiento de la normatividad que reclama la DO y la integración de los actores de la cadena de comercialización.

\section{REFERENCIAS}

Barrientos Felipa, P. (2015) La cadena de valor del Cacao en Perú y su oportunidad en el mercado mundial. Semestre Económico, 18(37), 129-156. https://doi.org/10.22395/seec.v18n37a5

Bianchi, E. \& Szpak C. (2013) Cadenas Globales de Producción: implicancias para el comercio internacional y su gobernanza. URL 
Biggeri, M., Ciani, F., \& Ferrannini, A. (2017). Aid effectiveness and multilevel governance: The case of a value chain development project in rural Ethiopia. The European Journal of Development Research, 29(4), 843-865. https://doi.org/10.1057/s41287-016-0064-1

Cava Jiménez, J. A., Millán Vásquez de la Torre, M. G., \& Hernández Rojas, R. (2019) Analysis of the Tourism Demand for Iberiam Ham Routes in Andalucía (Southern Spain): Tourist Profile. Sustainability, 11(16), 1-21. https://doi.org/10.3390/su11164278

Comisión Económica para América Latina y el Caribe. (2016). Cadenas de Valor. URL

Diario Oficial de la Federación (2012). Proyecto de Norma Oficial Mexicana PROY-NOM-189-SCFI2012, Chile habanero de la Península de Yucatán (Capsicum chinense Jacq.)Especificaciones y métodos de prueba. $\underline{\mathrm{URL}}$

Errázuriz Tortorelli, C. (2010) Indicaciones geográficas y denominaciones de origen: propiedad intelectual en progreso. Revista Chilena de Derecho, 37(2), 207-239. URL

Espejel Blanco, J. \& Fandos Herrera, C. (2009) Una aplicación del enfoque multiatributo para un producto agroalimentario con Denominación de Origen Protegida: el Jamón de Teruel. Estudios sociales (Hermosillo, Son.), 17(33), 136-161. URL

Fearne, A., Garcia Martinez, M., \& Dent, B. (2012). Dimensions of sustainable value chains: implications for value chain analysis. Supply Chain Management, 17(6), 575-581. https://doi.org/10.1108/13598541211269193

Flores Novelo, A., Jiménez Díez, O. \& Eastmond, A. (2008) Análisis comparativo de la estructura de los canales de distribución de chile habanero y miel en Yucatán. XII Congreso Anual de la Academia de las Ciencias Administrativas A.C. (ACACIA). URL

García Muñiz, A. S., \& Solís Arias, V. (2014). Comercio Internacional: cadenas Globales de Valor. Una aproximación desde la teoría de redes. Revista de Economía Mundial, (37), 151-180. $\underline{U R L}$

Gereffi, G. (2001). Las cadenas productivas como marco analítico para la globalización. Revista $\begin{array}{lll}\text { latinoamericana de economía, 32(125), } & \text { 9-37. }\end{array}$ http://dx.doi.org/10.22201/iiec.20078951e.2001.125.7389

González Andrade, S. (2015) Cadena de Valor económico del vino de Baja California México. Estudios fronterizos, 16(32) 163-193. URL

Granados Rojas, L. G. (2004). Indicaciones geográficas y denominaciones de origen. Un aporte para su Implementación en Costa Rica. $\underline{\text { URL }}$

Hernández Rojas, R. D. (2018) El turismo gastronómico en Andalucía: factores de Análisis. Revista Espacios, 39(22), 15. URL 
Kosacoff, B., López, A. \& Pedrazzoli, M. (2007). Comercio, inversión y fragmentación del mercado global: ¿está quedando atrás América Latina? $\underline{\mathrm{URL}}$

Kuijpers, R., \& Swinnen, J. (2016). Value Chains and Technology Transfer to Agriculture in Developing and Emerging Economies. American Journal of Agricultural Economics, 98(5), 1403-1418. https://doi.org/10.1093/ajae/aaw069

Los, B., Timmer, M. P., \& de Vries, G. J. (2015). How global are global value chains? A new approach to measure international fragmentation. Journal of Regional Science, 55(1), 6692. https://doi.org/10.1111/jors.12121

Maldonado Reyes, R. (2013). Caracterización y evaluación de tres poblaciones de chile habanero (Capsicum chínese Jacq.). (tesis de pregrado). URL

Martín Cerdeño, V. J. (2006) Denominaciones de origen: una revisión en el mercado alimentario español. Distribución y consumo, 16(90), 81-114. URL

Molano Pineda, E., Rico López, J., Tobón Palacio, A. M., Arbeláez Arango, T., Rendón Rincón, E., Correa Cadavid, C., Jiménez Osorio, A., González, S., \& Ceballos Ramírez, S. L. (2017) Significado y utilidad de la denominación de origen protegida -DOP- para los productores de loza del Carmen de Viboral. Science of Human, 2(2), 191-206. URL

Molina, M. S. (2015). La protección de la denominación de origen, indicación geográfica e indicación de procedencia. Estudio comparativo de las legislaciones de los Estados latinoamericanos y español. Revista Iberoamericana de Viticultura, Agroindustria y Ruralidad, 2(6), 46-69. URL

Mudambi, R., \& Puck, J. (2016). A Global Value Chain Analysis of the 'Regional Strategy' Perspective. Journal of Management Studies, 53(6), 1076-1093. https://doi.org/10.1111/joms.12189

Ocampo Thomason, P., Cocom Vazquez, J. M., \& Puc Canul, L. A. (2014) Diagnóstico histórico de la producción de chile habanero, papaya, plátano y miel en el sureste de México. URL

Moo Xix, F. J., Morales González, M. A., \& Canto Esquivel, A. M. (2016) Recursos capacidades de dos microempresas productoras de salsa de chile habanero. $21^{\circ}$ Encuentro Nacional sobre Desarrollo Regional en México. URL

Ríos, A. (26 de septiembre de 2014). Entrevista con José Apolinar Zapata-Aguilar. URL

Secretaría de Agricultura, Ganadería, Desarrollo Rural, Pesca y Alimentación (2012) Plan Rector del Sistema Producto Chile. $\underline{U R L}$

Stevens, C. (2001). Value Chains and Trade Policy the Case of Agriculture. IDS Bulletin, 32(3), 4659. https://doi.org/10.1111/j.1759-5436.2001.mp32003006.x 
Tamayo Manrique, J., Martínez y Ojeda, E., Monforte Méndez, G., Munguía Gil, A., \& Ruiz Martínez, A. (2014) La agroecología como propuesta de modelo de producción aplicado al cultivo de chile habanero en Peto, Yucatán. Revista Mexicana de Agronegocios, 35, 969978. $\underline{U R L}$

Trejo-Pech, C. O., López-Reyna, M. C., House, L. A., \& Messina, W. (2010). Appellation of Origin Status and Economic Development: A Case Study of the Mezcal Industry. International Food and Agribusiness Management Review, 13(2), 117-136. https://doi.org/10.22004/ag.econ.93346

Valenciano, J. de P. \& Román Sánchez, I. M. (2011) La importancia de la denominación de origen en la industria vitivinícola española. CIENCIA Ergo-Sum, 18(2), 135-144. URL 